\title{
The Design of User Database Resource Management System Based on Web
}

\author{
WU Ying ${ }^{1, a}$ \\ ${ }^{1}$ Department of Art Design, Wuhan Polytechnic, Wuhan 430074, China \\ awuying@126.com
}

\section{Keywords: Database Resource Management; Database System; Web}

\begin{abstract}
C} / \mathrm{S}$ mode to $\mathrm{B} / \mathrm{S}$ mode, the advantages of the software system based on WEB is more and more obvious: only use standard browser, of a lot of client configuration. However, late for a WEB application system of maintenance work, the development and maintenance staff still need to use database management system to operate, the server still exist many factors that affect efficiency. In this paper, the design and development is to attempt to solve the problem of a system, using web technology to manage user database resources, management of the database resources by local extend to any location, has realized the remote management of database system, and saves system maintenance for the related cost, providing a convenient for developers and maintenance personnel.
\end{abstract}

\section{Introduction}

The rapid development of information technology makes the entire community in all aspects of an information network of interconnected world, plus the rapid development and growing popularity of web technology met the traditional MIS had a huge impact. In this context, the angle $b$ and database technology combined with each other, they produce a web database. Combines the advantages of web database web technology and database technology, so that they have undergone a qualitative change. Web pages from static Web pages developed into a dynamic database-driven web pages, database development environment to achieve the separation and application environments [1-2].

With the development of web technology, distributed object technology and security technology, in order to achieve e-commerce on the Web, Web technology development challenges is how to achieve real-time transaction processing, scalability, scalability, security, and client authentication and so on. Web Object technology allows customers to direct contact with the relevant server, Web server to avoid the bottleneck formed by the distribution and object technology. And can be run from a single scalable server functionality to the entire server system to one or more servers to dynamically load balance client requests. These breakthrough technology can effectively solve the real-time transaction processing performed scalable Web. These technologies are in development, the site is the direction of Web database development.

\section{The related theory and technology analysis}

Database System is a system of databases and their corresponding database management software made. DBS is a data processing to meet the needs and developed an ideal core body of data processing, is an actual running of storage, maintenance and application software systems to provide data, storage media, the processing and management system the aggregates. A database management system for normal operation of the database is to provide the necessary protection [3]. For most users, the database management system is the only channel for interaction between the user and the database. When the application requires the use of a storage medium to store data, you cannot directly read the data, you must apply for access to the database through the database management software, followed by the database management system to retrieve the relevant data by the application user actions Program use, the interface is a database management system software application and the required data between the application data. 
In database systems engineering, architecture design data warehouse is a key link. Data warehouse project to be achieved many goals, such as improving the quality of service, reduce operating costs, accelerate the development of new products and services varieties to enhance their efficiency in response to the competitive environment, they have to reflect the reasonable and feasible to implement a data warehouse system structural design, and data access is the core problem of the entire data warehouse architecture [4-5]. Data warehouse technology essence, is an information integration technology. Data warehouse from multiple information sources to obtain the original data, after finishing, stored in an internal database data warehouse by providing users with access to tools, to provide a unified data warehouse user, coordinated and integrated information environment to support business global decision-making process in depth and comprehensive analysis of business management. To achieve this goal, a data warehouse in general consists of the following seven major components:

Data warehouse architecture shown in Figure 1.

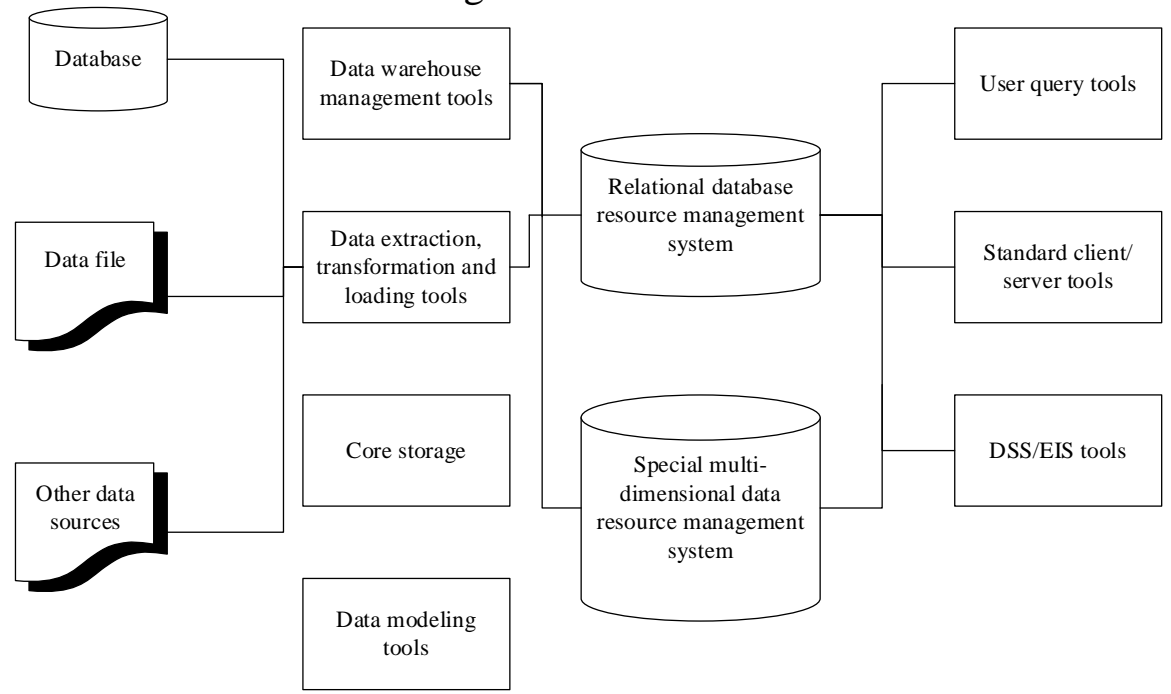

Figure 1. Data warehouse architecture

Data source. Source data for data warehousing, such as various production systems database, online transaction processing system operational data, external data source and so can be used as a data warehouse data source.

Data extraction, transformation and loading tools. Its function is to extract the data from the data source, data inspection and sorting, and according to the design requirements of the data warehouse, the data are re-organizing and processing, and loaded into the data warehouse target database.

Data modeling tools. The source and target databases used to build the data warehouse information model.

Core Storage. Stores data models and metadata. Wherein the metadata describes the data warehouse information source and target data itself, the definition of the data conversion process from the source to the target data.

Data Warehouse target database. Upon examination storage, sorting, processing and re-organize the data after. As a data warehouse target database that you can use traditional relational database management system, you can also use special multidimensional database management system.

Front-end data access and analysis tools. For business analysis and decision-makers to access data in the target database, and further in-depth analysis. Such tools currently on the market that can be obtained are: relational query tools, standard client / server tools and decision support systems (DSS) / Chief Information Systems (EIS) software package. 7. Data Warehouse Management tools: Provides management tools for running a data warehouse, including content security management, storage management, and other aspects. 


\section{User database resource management system requirements analysis}

In this paper, the system needs analysis, summarized the user database management system resource classes and objects, and to analyze the relationship between planning from the status quo and the part of the business processes, based on the needs analysis, the system data flow describe the overall framework of the system design, and then the system is divided into different functional modules, based on the basic principles of database design, the main structure of the database design [6]. WEB-based DBMS through the research and development, the full realization of resource management flexibility to enhance the user database, you can remotely manage WEB end user database resources controlled by the server no longer need for cumbersome maintenance; DBMS by state monitoring, network and intelligent database operations to achieve the service management capabilities, database management, data management function table, view management, command-line tool control functions, the system function business processes. System function module structure shown in Figure 2.

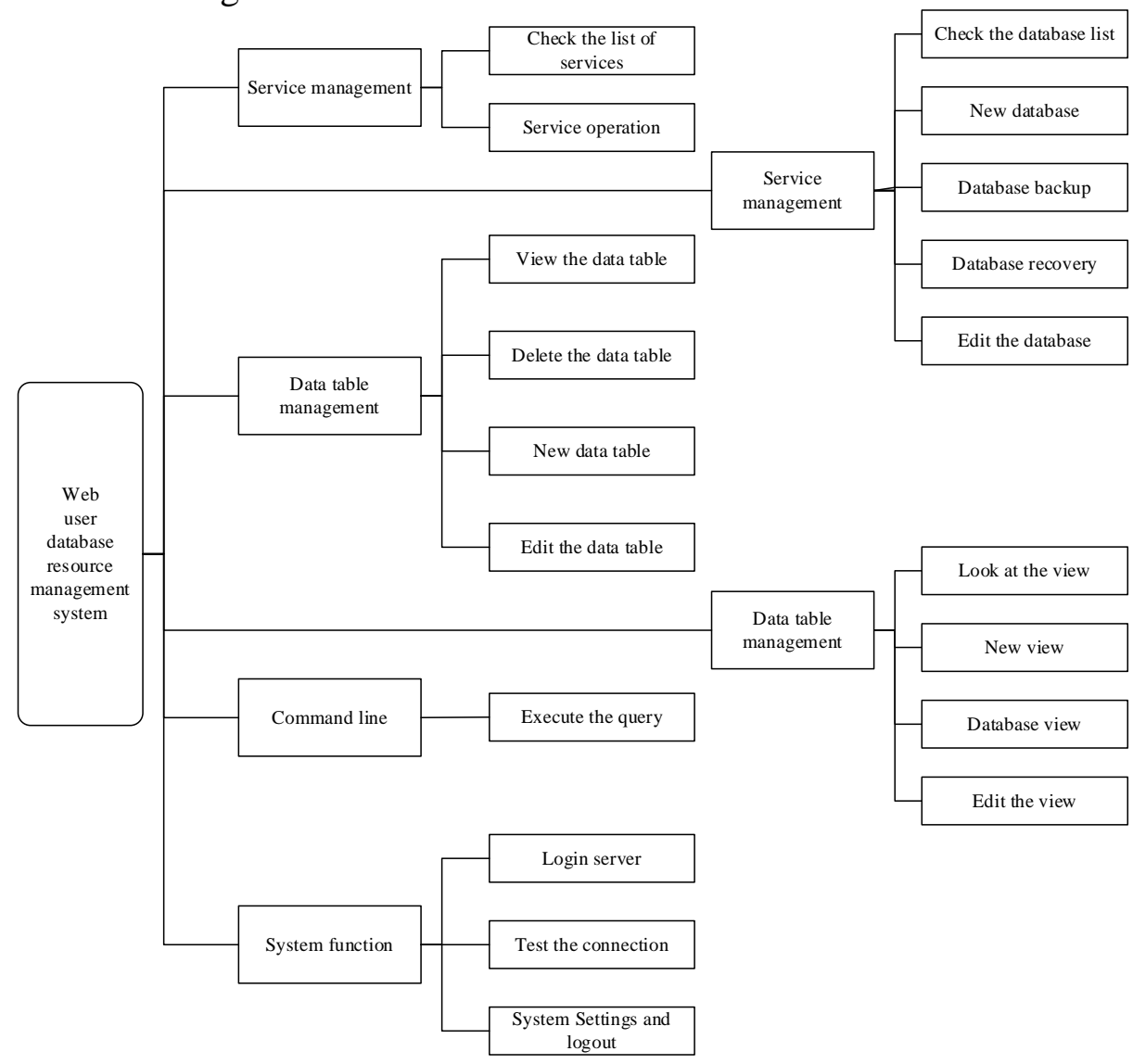

Figure 2. System function module structure

The most important architectural approach to system development process is a ring system, a stable and efficient software systems, both to meet the needs of users on the functional use, but at a relatively efficient architecture platforms to achieve, which also post-secondary system maintenance and development to provide favorable conditions. WEB-based user database resource management system using three-tier approach to the MVC architecture design and implementation at all levels of responsibility and function is very clear, which greatly improves the scalability and maintainability, and reusability of system components developed with a strong, Components developed universal strong for post-transplant to other management systems provide a convenience. Design of the system development reflects the separation of interface and implementation of programming ideas, dependencies between client and system components is greatly reduced, enhanced design flexibility, the underlying design and implementation of the adjustment will not affect the upper produce significant changes. 


\section{Database resource management system design}

By designing the user database resource management system based on the realization of the WEB, users can log on to a different database server, select different authentication methods and logged in user, implementation-dependent operation of the database, data tables, data view. In addition, because C \# for SQL Server syntax for the various versions of SQL operations are relatively uniform, so the system can manage different versions of the SQL Server database, and database resources to achieve remote management WEB side. Database resource management system design flow shown in Figure 3.

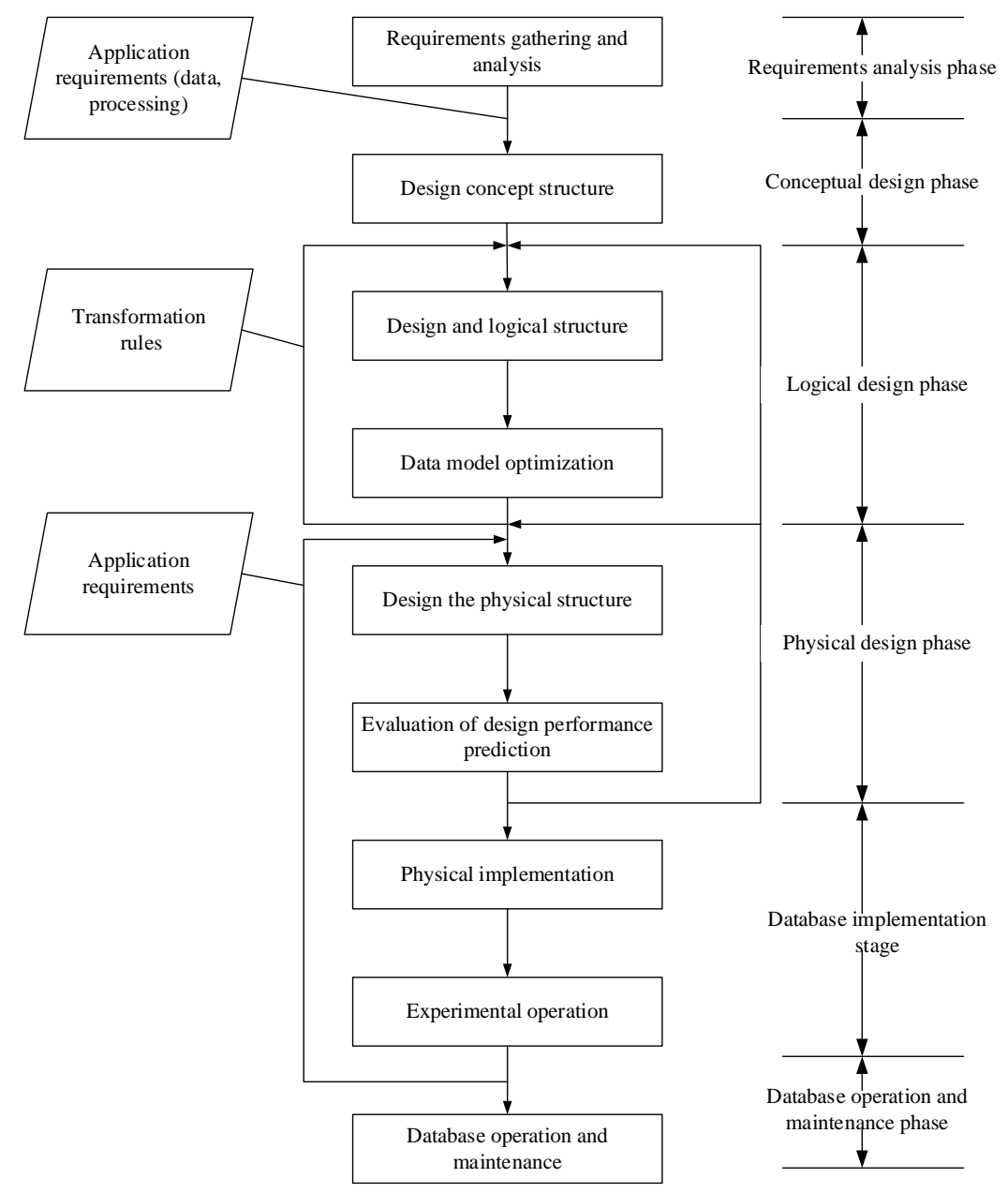

Figure 2. Database resource management system design

In the user database resource management system based on the WEB, implements database service management, database management, data management table, view, manage, command line tools and system utilities settings and other functions for maintenance of the database for the system administrator to improve efficiency. Case database system maintenance personnel require background data maintenance, eliminating the need for cumbersome to deploy and maintain server-side through the background, but like using ordinary-based Browser / Server mode of WEB applications like databases for related operations. Based on the concept of the system, most of the development direction of modern database management system with new ideas, and that is from the traditional desktop applications (DBMS) to WEB application (WEB-based database resource management system) transition. In summary, the study and implementation of the database resource management system on the one hand has a high research value and practical value, it also has a relatively high innovation. 


\section{Conclusion}

With the rapid development of Internet, the degree of integration of database technology and network technology has become increasingly close, network application development model by C/S structure to B/S structure changes, how to build a stable and efficient WEB-based user database resource management system has become the research field of information technology is an important direction. Using B / S architecture mode, make full use of WEB server functionality using standard WEB-based user interface to control and manage database resources, interactive way to achieve the operating system platforms and databases across categories, remote management database resources can be achieved, user management database resources for more convenient. In this paper, the architecture of the web database systems, Web database access techniques were studied, designed a specific web database resource management system integration solutions.

\section{Reference}

[1] Nurmi D, Wolski R, Grzegorczyk C, et al. The eucalyptus open-source cloud-computing system[C]//Cluster Computing and the Grid, 2009. CCGRID'09. 9th IEEE/ACM International Symposium on. IEEE, 2009: 124-131.

[2] Coronel C, Morris S, Rob P. Database systems: design, implementation, and management[M]. Cengage Learning, 2009.

[3] Harris P A, Taylor R, Thielke R, et al. Research electronic data capture (REDCap)—a metadata-driven methodology and workflow process for providing translational research informatics support[J]. Journal of biomedical informatics, 2009, 42(2): 377-381.

[4] Davies D K, Ilavajhala S, Wong M M, et al. Fire information for resource management system: archiving and distributing MODIS active fire data[J]. Geoscience and Remote Sensing, IEEE Transactions on, 2009, 47(1): 72-79.

[5] Buyya R, Yeo C S, Venugopal S, et al. Cloud computing and emerging IT platforms: Vision, hype, and reality for delivering computing as the 5th utility[J]. Future Generation computer systems, 2009, 25(6): 599-616.

[6] Miller M A, Pfeiffer W, Schwartz T. Creating the CIPRES Science Gateway for inference of large phylogenetic trees[C]//Gateway Computing Environments Workshop (GCE), 2010. IEEE, 2010: 1-8. 